Laser Chem. 1988, Vol. 9, pp. 359-368

(C) 1988 Harwood Academic Publishers GmbH

Photocopying permitted by license only

Reprints available directly from the Publisher

Printed in the United Kingdom

\title{
Laser-Induced-Fluorescence Spectrum of the CNN Molecule
}

\author{
M. C. CURTIS, A. P. LEVICK and P. J. SARRE \\ Department of Chemistry, University of Nottingham, University Park, \\ Nottingham NG7 2RD, UK
}

(Received 20 May, 1988)

\begin{abstract}
We have recorded a laser excitation spectrum of the $A^{3} \Pi-X^{3} \Sigma^{-}$electronic band system of the CNN radical in the gas phase, at a resolution of $c a .0 .8 \mathrm{~cm}^{-1}$. The rotational branch structure of the vibrational band near $419 \mathrm{~nm}$ has been simulated and molecular parameters are obtained. The results are compared with data from laser-inducedfluorescence spectra of CNN trapped in a low-temperature matrix. A revised value for the spin-orbit parameter in the $A^{3} \Pi$ state is obtained and comparison is made with the values for related molecules. The possible detection of CNN in astrophysical sources is considered and the general features of its rotational spectrum in the ground electronic state are described.
\end{abstract}

KEY WORDS: Laser, CNN, fluorescence, electronic, spectrum.

\section{INTRODUCTION AND REVIEW}

Electronic spectra of triatomic molecules have been known for many years. ${ }^{1}$ Of those containing mixed combinations of carbon and nitrogen atoms, rotational analyses of the lowest electronic band systems of $\mathrm{NCN}^{2}, \mathrm{CCN}^{3}$ and $\mathrm{CNC}^{4}$ have been published. In this paper we report the detection of a laser-induced-fluorescence spectrum of the CNN molecule in the gas phase at a resolution of $c a .0 .8 \mathrm{~cm}^{-1}$. The spectrum was discovered accidentally while searching for a spectrum of the $\mathrm{Si}_{2}$ molecule.

To the best of our knowledge there has been no published rotational analysis of a gas-phase electronic spectrum of $\mathrm{CNN}$, although the 
recording of a spectrum was mentioned briefly in a paper published in 1969. ${ }^{5}$ In this work, ${ }^{5}$ absorptions at $419 \mathrm{~nm}$ and $397 \mathrm{~nm}$ were observed at low resolution following flash photolysis of carbon suboxide in the presence of nitrogen. The absorptions were identified as originating from CNN in part by comparison with spectra of CNN obtained in low-temperature matrices. The absorption at $419 \mathrm{~nm}$ is at the same wavelength as found in our laser excitation spectrum. It is of interest to note that an unidentified feature at $419.8 \mathrm{~nm}$ which was reported in $1963^{6}$ in the chemiluminescent emission from a carbon tetrachloridepotassium flame may also have been due to $\mathrm{CNN}$.

Whereas the gas-phase electronic spectrum of $\mathrm{CNN}$ is not well characterised, the CNN molecule has been studied extensively in matrices at low temperatures; electronic, ${ }^{7-15}$ vibrational ${ }^{10-12,16}$ and electron spin resonance spectra ${ }^{17,18}$ have been reported. The first paper of direct relevance to the work described here is that of Robinson and McCarty. ${ }^{7}$ They observed an absorption at $424.0 \mathrm{~nm}$ following photolysis of diazomethane in a krypton matrix and attributed the feature tentatively to $\mathrm{CNN}$ or $\mathrm{HCN}_{2}$. Similar absorptions at $418.2 \mathrm{~nm}$ and $396.8 \mathrm{~nm}$ were observed at the same time by Goldfarb and Pimentel. ${ }^{8}$ Further studies of electronic spectra in lowtemperature matrices include the observation of absorptions at $418.9 \mathrm{~nm}$ and $396.4 \mathrm{~nm}$ recorded following photolytic production of carbon atoms which reacted with $\mathrm{N}_{2} \cdot{ }^{11}$ These were assigned to $\mathrm{CNN}$ in a combined ultraviolet/infra-red experiment. ${ }^{11}$ It has also been shown that the same absorption feature at $c a .420 \mathrm{~nm}$ is obtained by the reaction of vaporised carbon with $\mathrm{N}_{2}$ in a matrix. ${ }^{9}$ Two further papers on the electronic spectroscopy of CNN in matrices were published in 1977. Wilkerson and Guillory reported emission spectra, excitation frequencies and single vibronic state lifetimes ${ }^{13}$ and comparison of their data with that of the known isoelectronic molecules NCN and $\mathrm{CCO}$ led them to conclude that the symmetry of the excited state in the observed transitions was ${ }^{3} \Pi$. In the same year, Bondybey and English ${ }^{14}$ described laser-induced-fluorescence and dispersed emission spectra of CNN, in addition to a study of vibrational relaxation. They concluded that the symmetry of the electronic transition was probably ${ }^{3} \Pi-{ }^{3} \Sigma^{-}$and measured the excited state vibrational frequencies. ${ }^{14}$ The dye laser excitation spectrum ${ }^{14}$ of the $(0,0,0)-(0,0,0)$ band in an argon matrix exhibits three closely spaced peaks near $23,597 \mathrm{~cm}^{-1}$ which were attributed ${ }^{14}$ to excitations to the three spin-orbit components of 
the upper electronic state of the transition. A value of $9 \mathrm{~cm}^{-1}$ was deduced for the spin-orbit parameter in the ${ }^{3} \Pi$ state of $\mathrm{CNN}$ in solid argon. The most recently published matrix study concerns a different electronic transition observed between $190 \mathrm{~nm}$ and $260 \mathrm{~nm}$ which has been assigned to ${ }^{3} \Sigma^{-}-{ }^{3} \Sigma^{-}$transition. ${ }^{15}$ In addition to this experimental work, calculations of the electronic structure,${ }^{19}$ bond lengths ${ }^{19}$ and spin-spin interaction constant ${ }^{20}$ for the $X^{3} \Sigma^{-}$state have been reported.

\section{EXPERIMENTAL}

The apparatus employed has been used extensively for studies of the high-resolution spectroscopy of the Swan bands of the $\mathrm{C}_{2}$ molecule. ${ }^{21-23}$ It consists of a stainless steel six-way cross to which a reservoir is attached at the bottom, usually containing sodium metal. In normal use the cell is heated to generate sodium vapour and reactant gases are introduced through a nozzle at the bottom of the reservoir. A gas flow of nitrogen is used to carry the reactant vapour through the cell region and also to prevent sodium vapour from condensing on the cell windows. At the time of the CNN detection, vapour of $\mathrm{SiBr}_{4}$ was flowing into the sodium vapour in an attempt to detect one of the band systems of $\mathrm{Si}_{2}$ through reactions analogous to the formation of $\mathrm{C}_{2}{ }^{21}$ After some hours the spectrum in Figure 1 appeared. Clearly the chemistry as planned would not be expected to generate the CNN molecule and the identification of this molecule as the carrier of the spectrum is based on other considerations. It was found that the needle valve controlling the flow of $\mathrm{SiBr}_{4}$ had been eroded away by reaction. It is possible that the $\mathrm{CNN}$ was generated by a reaction on the surface of the needle valve in a manner similar to the mechanism by which it has been formed in matrix isolation studies, ${ }^{9}$ although the role of residual carbon on the surface of the cell itself (from previous work on $\mathrm{C}_{2}$ ) cannot be ruled out. Exploration of more controlled methods for the production of $\mathrm{CNN}$ is in progress.

A Coherent CR-590 broad-band standing wave c.w. laser operating with Stilbene 3 dye was employed in this work. Up to $500 \mathrm{~mW}$ of radiation with a bandwidth of $c a .0 .8 \mathrm{~cm}^{-1}$ and tunable between $406 \mathrm{~nm}$ and $476 \mathrm{~nm}$ was obtained when the dye laser was pumped with $3 \mathrm{~W}$ of ultraviolet radiation from an argon ion laser. The laser beam was chopped mechanically at $3 \mathrm{kHz}$ and lock-in detection of the fluorescence signal was employed. 


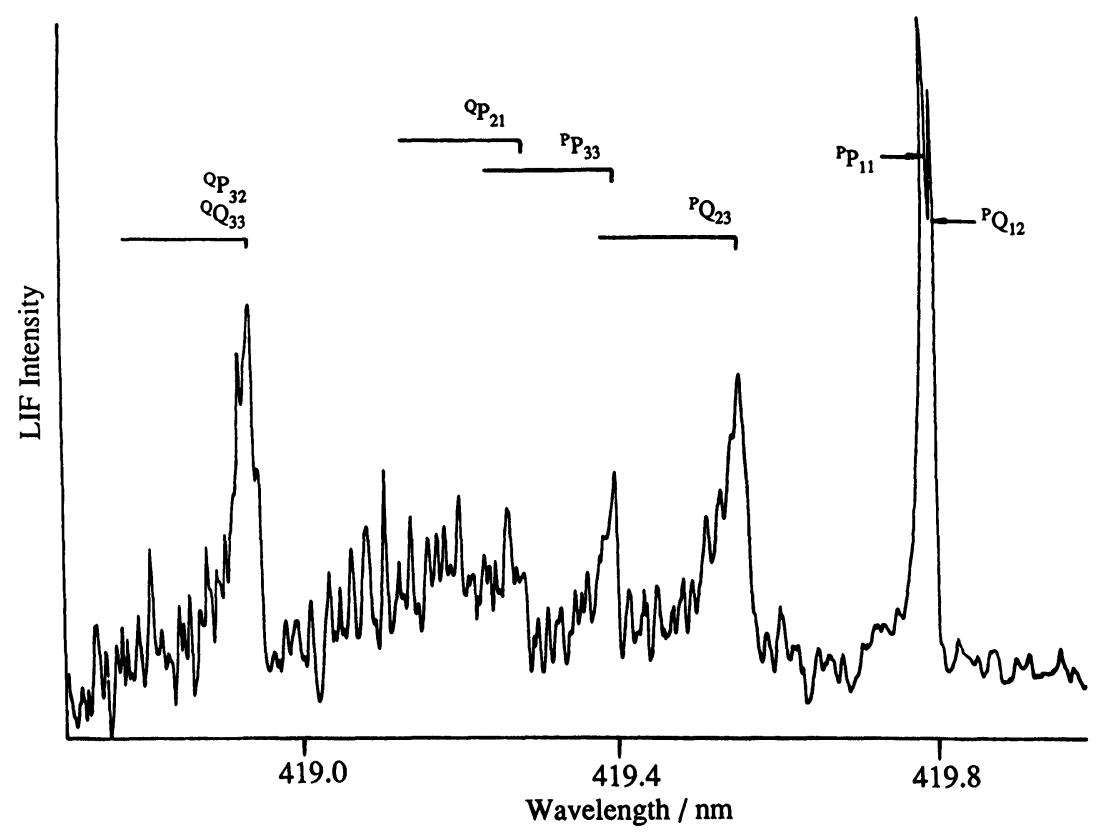

Figure 1 Experimental laser-induced fluorescence spectrum of CNN.

\section{SPECTROSCOPIC ANALYSIS AND SIMULATION}

The overall appearance of the spectrum in Figure 1 is of a vibrational band comprised of a series of violet-degraded rotational branches. The long-wavelength end of the band is marked by a narrow intense feature which is split into a doublet. This feature also appears to be slightly degraded to the blue. In addition there are at least three less intense and more open branches which exhibit rotational structure with line separations of about $0.8 \mathrm{~cm}^{-1}$. There is a narrow feature about $48 \mathrm{~cm}^{-1}$ to the blue of the strongest doublet, and about $145 \mathrm{~cm}^{-1}$ further to the blue are two very weak features also separated by $48 \mathrm{~cm}^{-1}$, the first of which shows a doublet splitting analogous to its probable counterpart in the $419 \mathrm{~nm}$ band. The spacing of $0.8 \mathrm{~cm}^{-1}$ in parts of the spectrum suggests a rotational constant of $c a .0 .4 \mathrm{~cm}^{-1}$ if the carrier of the spectrum is a linear triatomic carbon/nitrogencontaining molecule with approximately the same geometry in the two 
electronic states. The spectrum does not correspond to that of any known gas-phase molecule in the literature, but the origin of the transition occurs close to that of the firmly identified matrix spectrum of $\mathrm{CNN}^{11,13,14}$ and the low-resolution gas-phase observation. ${ }^{5}$ Furthermore, a rotational constant of about $0.4 \mathrm{~cm}^{-1}$ is expected for CNN in comparison with an ab-initio calculation of the bond lengths for $\mathrm{CNN}^{19}$ and experimental work on similar molecules; for example the NCN molecule has a B value of $0.3968 \mathrm{~cm}^{-1}$ in its ground electronic state. ${ }^{2}$ The resolution of our spectrum is limited by the laser bandwidth and this precludes a measurement of the wavenumbers of the individual rotational lines. However, the spectrum is of sufficiently good quality that a simulation of the band can be undertaken.

The first step was to take the symmetries of the ground and excited electronic states to be ${ }^{3} \Sigma^{-}$and ${ }^{3} \Pi$. The electron spin resonance studies $^{17,18}$ have shown that the ground state is a triplet and the molecule is expected to be linear in both ground and excited states according to the molecular orbital arguments of Walsh. ${ }^{24}$ The ground state arises from the electronic configuration ... $(6 \sigma)^{2}(1 \pi)^{4}(7 \sigma)^{2}(2 \pi)^{2}$ which gives rise to states of ${ }^{3} \Sigma^{-},{ }^{1} \Delta$ and ${ }^{1} \Sigma^{+}$symmetry. Two electronic states, ${ }^{1} \Pi$ and ${ }^{3} \Pi$, arise from the first excited configuration in which one of the $7 \sigma$ electrons is promoted to the $2 \pi$ orbital and, in common with other workers, we assign the upper state of the transition as ${ }^{3} \Pi$ and the vibrational band near $419 \mathrm{~nm}$ to be $(0,0,0)-(0,0,0)$. We used published expressions for the energy levels of the $X^{3} \Sigma^{-25}$ and $A^{3} \Pi^{26}$ states to calculate the rotational levels and hence the transitions. The allowed electric dipole transitions which give rise to the rotational branches have been illustrated by Herzberg. ${ }^{27}$ As a test of the program, line positions for the corresponding spectra of the isoelectronic $\mathrm{CCO}$ and NCN molecules were calculated using the published parameters and were found to be in good agreement with the known transition frequencies. Formulae for the rotational line strengths have been published ${ }^{28}$ and these were used to compute the intensities. It was found that these expressions were satisfactory in reproducing the observed intensities in the spectra of $\mathrm{CCO}$ and $\mathrm{NCN}$.

It was necessary to make initial estimates for the molecular parameters of CNN. The published parameters for $\mathrm{CCO}$ and NCN are reproduced in Table I and were used as a guide. In the simulation, we varied the spin-orbit parameter $A$ and the rotational constants, $B^{\prime}$ and $B^{\prime \prime}$, whereas the values for the spin-spin $\lambda$, spin-rotation $\gamma$ and centrifu- 
Table I Experimental parameters for $\mathrm{CCO}, \mathrm{NCN}$ and $\mathrm{CNN}$, and the parameters used in the simulation of the laser-induced-fluorescence spectrum of CNN.

\begin{tabular}{lllll}
\hline Parameter $^{\mathrm{a}}$ & $\mathrm{CCO}^{29}$ & $\mathrm{NCN}^{2}$ & $\mathrm{CNN}^{\mathrm{b}}$ & $\mathrm{CNN}$ (this work) \\
\hline$X^{3} \Sigma^{-}$ & & & & \\
$B / \mathrm{cm}^{-1}$ & 0.3851 & 0.3968 & & $0.4136(100)^{\mathrm{c}}$ \\
$\lambda / \mathrm{cm}^{-1}$ & 0.386 & 0.783 & $0.585^{18}$ & $0.585^{\mathrm{d}}$ \\
$\gamma / \mathrm{cm}^{-1}$ & -0.0007 & -0.001 & & $-0.0007^{\mathrm{e}}$ \\
$A^{3} \Pi$ & & & & \\
$B / \mathrm{cm}^{-1}$ & 0.4066 & 0.3962 & & $0.4250(100)^{\mathrm{c}}$ \\
$A / \mathrm{cm}^{-1}$ & -35.36 & -37.56 & $9^{14}$ & $-26.5^{\mathrm{c}}$ \\
$T / \mathrm{cm}^{-1}$ & 11650.8 & 30383.74 & $23597^{14}$ & $23850^{\mathrm{c}}$ \\
\hline
\end{tabular}

a All parameters refer to the $(0,0,0)$ vibrational level.

b Previous experimental work.

c The values quoted are those used in the simulation (see Figure 2) and the uncertainties are estimates only. The simulation is far more sensitive to the difference in the rotational constants than their absolute values. We obtain $B^{\prime}-B^{\prime \prime}=0.0114(10) \mathrm{cm}^{-1}$.

d The value for $\lambda$ assumes $\lambda=2 \mathrm{D}$.

e Value constrained to that given in ${ }^{29}$.

gal distortion parameters, $D^{\prime}$ and $D^{\prime \prime}$, were held constant. The two strongest features of the spectrum are the ${ }^{{ }} P_{32} /{ }^{Q} Q_{33}$ and ${ }^{P} Q_{12} /{ }^{P} P_{11}$ branch heads and their separation in $\mathrm{cm}^{-1}$ is equal to approximately twice the spin-orbit parameter in the $A^{3} \Pi$ state. In CCO and NCN the separation is about $70 \mathrm{~cm}^{-1}$ while in CNN we find that it is only about $48 \mathrm{~cm}^{-1}$, so the spin-orbit parameter is significantly smaller in CNN. As the band is degraded to the violet, it follows that the rotational constant is larger in the excited state than in the ground state, but the ground and excited state values can both be expected to fall in the region of $0.4 \mathrm{~cm}^{-1}$. The spin-spin interaction in the ground state is expected to have a significant effect on the spectrum. Fortunately, a good estimate for $\lambda$ can be obtained from the electron spin resonance studies in a low-temperature matrix ${ }^{17,18}$ and using the relationship that the zero field splitting parameter, $D$, is equal to twice $\lambda$. This works well for NCN and CCO when the electron spin resonance values for $D$ are compared with the gas-phase values of $\lambda .{ }^{18} \mathrm{We}$ therefore take the value of $1.17 / 2=0.585 \mathrm{~cm}^{-1}$ for $\lambda$ in the simulation of our spectrum of CNN. The centrifugal distortion constants, $D^{\prime}$ and $D^{\prime \prime}$, and the spinrotation parameter in the ground state were constrained to the values 
for $\mathrm{CCO}^{29}$ A rotational temperature of $500 \mathrm{~K}$ and a laser bandwidth of $0.8 \mathrm{~cm}^{-1}$ were used in the computation.

The best simulation achieved after variation of $B^{\prime \prime}, B^{\prime}$ and $A$ is shown in Figure 2 and the parameters determined and employed in the simulation are given in Table I. The general features of the spectrum are well reproduced with only a few discrepancies in the relative intensities of some of the rotational branches. In order to achieve a good reproduction of the observed spectrum, it was necessary to adopt a value for the spin-orbit parameter in the $A^{3} \Pi$ state of $-26.5 \mathrm{~cm}^{-1}$. This is markedly different from the value deduced from the spectrum in an argon matrix ${ }^{14}$ from which a value of $9 \mathrm{~cm}^{-1}$ was obtained. However our result is much closer to the values for $\mathrm{CCO}$ and NCN in their $A^{3} \Pi$ states which have values of $-35.36 \mathrm{~cm}^{-1}$ and $-37.56 \mathrm{~cm}^{-1}$ respectively. Our result also follows the general trend for isoelectronic molecules as shown in Table II. The values for the rotational constants

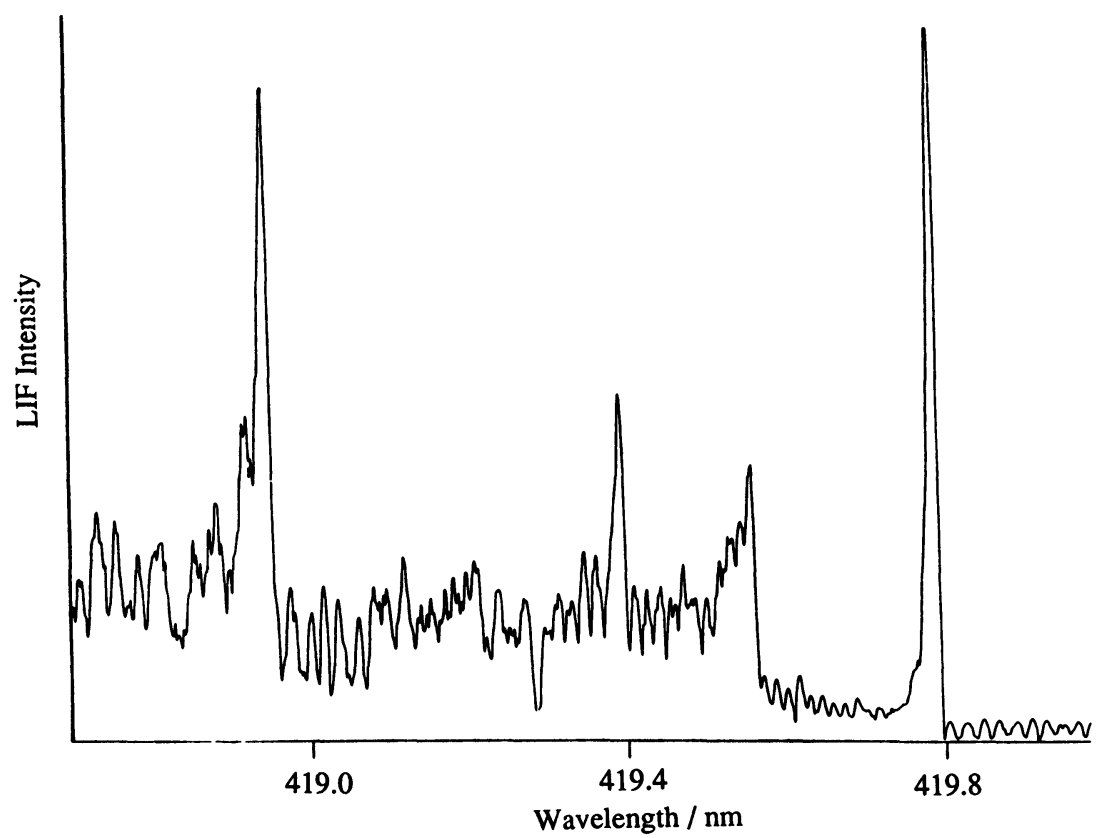

Figure 2 Simulation of the laser-induced fluorescence spectrum of CNN. The parameters employed in the generation of this figure are discussed in the text and listed in Table I. 
Table II Values for the spin-orbit parameters for related and isoelectronic triatomic molecules with $\Pi$ electronic states.

\begin{tabular}{lllll}
\hline Molecule & State & Electronic configuration & $A / \mathrm{cm}^{-1}$ & Ref. \\
\hline CCN & $X^{2} \Pi$ & $\ldots \ldots(2 \pi)^{1}$ & 40.34 & 3 \\
CNC & $X^{2} \Pi$ & $\ldots \ldots(2 \pi)^{1}$ & 26.41 & 4 \\
NCN & $A^{3} \Pi$ & $\ldots \ldots(2 \pi)^{3}$ & -37.56 & 2 \\
CNN & $A^{3} \Pi$ & $\ldots \ldots(2 \pi)^{3}$ & -26.5 & This work \\
CCO & $A^{3} \Pi$ & $\ldots \ldots(2 \pi)^{3}$ & -35.36 & 29 \\
NNN & $X^{2} \Pi$ & $\ldots \ldots(2 \pi)^{3}$ & -71.3 & 30 \\
NCO & $X^{2} \Pi$ & $\ldots \ldots(2 \pi)^{3}$ & -95.59 & 31 \\
\hline
\end{tabular}

in the electronic states are reasonable, although the simulation is not very sensitive to their absolute magnitudes. On the other hand, their relative size has a marked effect on the spectrum and the increase in rotational constant on excitation follows the behaviour for the isoelectronic CCO molecule. In summary we have shown that the $419 \mathrm{~nm}$ band is simulated very well assuming that the upper state has ${ }^{3} \Pi$ symmetry, taking reasonable values for the rotational constants and with a value for the spin-orbit parameter of $c a .-26.5 \mathrm{~cm}^{-1}$.

\section{CNN AS AN EXTRATERRESTRIAL MOLECULE}

Part of the motivation for this work is provided by the possible existence of the CNN molecule in interstellar gas clouds and circumstellar shells. Numerous related molecules have been detected in these sources by radioastronomy and the electric dipole moment of CNN is calculated to have a reasonably high value of 1.022 Debye.${ }^{19}$ Using the molecular parameters obtained from the electron spin resonance spectrum and our simulation, we have calculated approximate rotational energy levels for $\mathrm{CNN}$ in the ground electronic state. These, together with the allowed transitions, are shown in Figure 3. The energy levels are those of a molecule in $\mathrm{a}^{3} \Sigma^{-}$electronic state and were calculated using the formulae given in Ref. 25 . Given the uncertainty in the molecular parameters, these predictions cannot be taken as more than a guide but we hope that they may be of some assistance in identifying a few of the unidentified lines recorded by radioastronomers. Only those transitions falling below $80 \mathrm{GHz}$ are included. For 

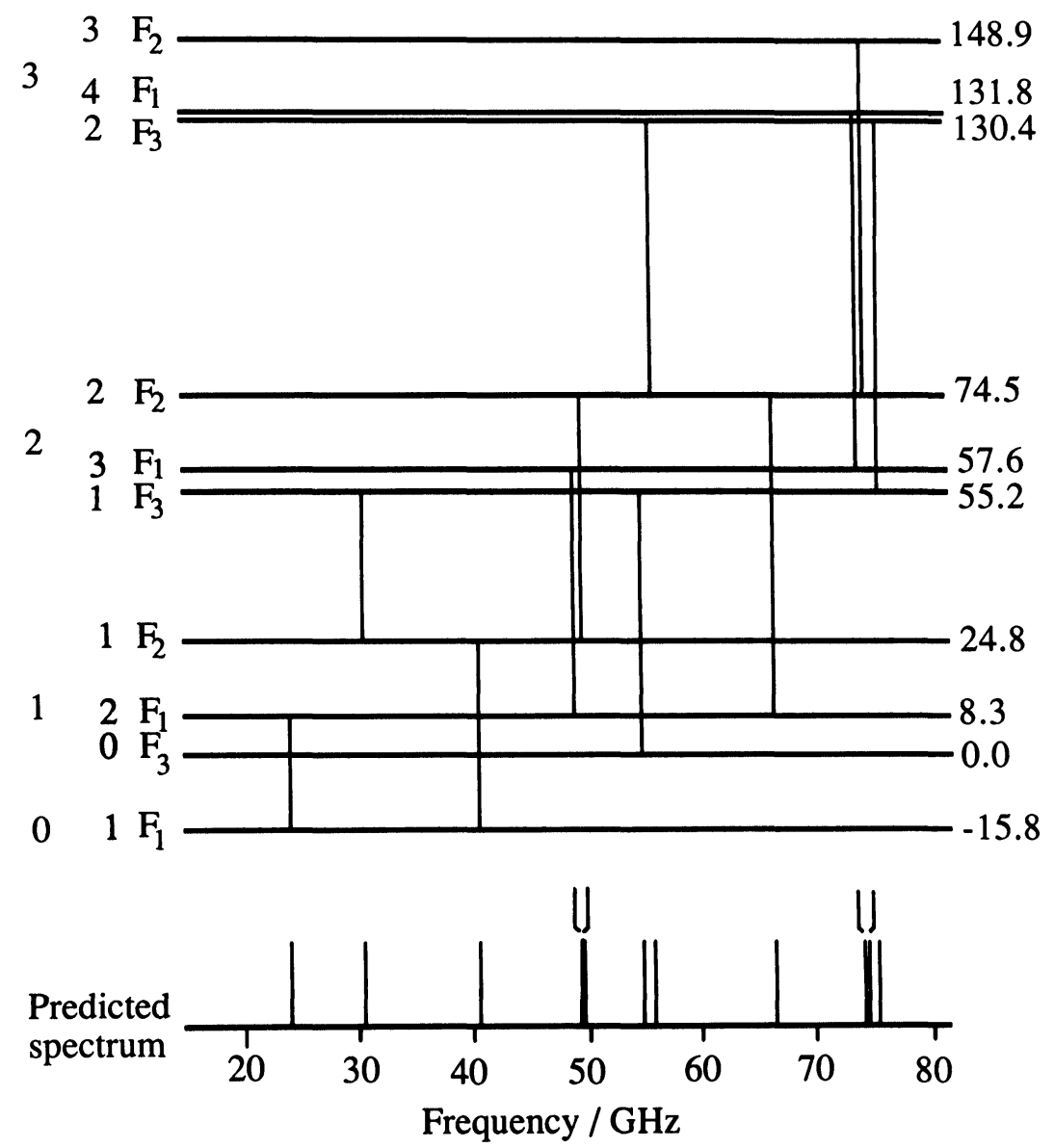

Figure 3 Calculated energy levels and rotational transitions in the $X^{3} \Sigma^{-}$state of the CNN molecule.

$\mathrm{CNN}$, the spin-spin parameter is comparable in magnitude to the rotational constant so the simple relationship between successive rotational transitions which occurs for other linear molecules does not hold for most of the transitions. Exceptions to this are the $\Delta J=+1$, $\Delta N=+1$ transitions involving the $F_{2}$ levels which occur at intervals of 
almost exactly twice the value of the rotational constant. Many of the lines in the spectrum of $\mathrm{CNN}$ are expected to carry characteristic hyperfine patterns arising from interactions due to the nuclear spin of the nitrogen nuclei.

\section{Acknowledgements}

We thank the SERC, the Research Corporation Trust and the Nuffield Foundation for research grants and Coherent (UK) Ltd. for support under the CASE scheme. PJS thanks the Nuffield Foundation for a Science Research Fellowship. MCC and APL thank the SERC for research studentships.

\section{References}

1. G. Herzberg, Molecular Spectra and Molecular Structure III. Electronic Spectra and Electronic Structure of Polyatomic Molecules, van Nostrand, New York, 1966.

2. G. Herzberg and D. N. Travis, Can. J. Phys. 42, 1658 (1964).

3. A. J. Merer and D. N. Travis, Can. J. Phys. 43, 1795 (1965).

4. A. J. Merer and D. N. Travis, Can. J. Phys. 44, 353 (1966).

5. W. Braun, A. M. Bass, D. D. Davis and J. D. Simmons, Proc. Roy. Soc. Lond. A312, 417 (1969).

6. W. J. Miller and H. B. Palmer, 9th International Symposium on Combustion (Cornell, 1963) p. 90.

7. G. W. Robinson and M. McCarty, Jr., J. Am. Chem. Soc. 82, 1859 (1960).

8. T. D. Goldfarb and G. C. Pimentel, J. Am. Chem. Soc. 82, 1865 (1960).

9. W. Weltner, Jr. and D. McLeod, Jr., J. Chem. Phys. 40, 1305 (1964).

10. D. E. Milligan, M. E. Jacox and A. M. Bass, J. Chem. Phys. 43, 3149 (1965).

11. D. E. Milligan and M. E. Jacox, J. Chem. Phys. 44, 2850 (1966).

12. W. Weltner, Jr. and D. McLeod, Jr., J. Chem. Phys. 45, 3096 (1966).

13. J. L. Wilkerson and W. A. Guillory, J. Molec. Spectrosc. 66, 188 (1977).

14. V. E. Bondybey and J. H. English, J. Chem. Phys. 67, 664 (1977).

15. M. E. Jacox, J. Molec. Spectrosc. 72, 26 (1978).

16. R. L. DeKock and W. Weltner, Jr., J. Am. Chem. Soc. 93, 7106 (1971).

17. E. Wasserman, L. Barash and W. A. Yager, J. Am. Chem. Soc. 87, 2075 (1965).

18. G. R. Smith and W. Weltner, Jr., J. Chem. Phys. 62, 4592 (1975).

19. C. Thomson, J. Chem. Phys. 58, 841 (1973).

20. G. R. Williams, Chem. Phys. Lett. 25, 602 (1974).

21. M. C. Curtis and P. J. Sarre, J. Chem. Soc. Chem. Comm. 604 (1986).

22. M. C. Curtis and P. J. Sarre, J. Mol. Spectrosc. 114, 427 (1985).

23. M. C. Curtis and P. J. Sarre, Molec. Phys. (in the press).

24. A. D. Walsh, J. Chem. Soc. 2266 (1953).

25. R. Schlapp, Phys. Rev. 51, 342 (1937).

26. A. Budo, Z. Physik. 105, 579 (1937).

27. G. Herzberg, Molecular Spectra and Molecular Structure I. Spectra of Diatomic Molecules, van Nostrand, New York (1950).

28. I. Kovacs, Rotational Structure in the Spectra of Diatomic Molecules, Amer. Elsevier, New York, 1969.

29. C. Devillers and D. A. Ramsay, Can. J. Phys. 49, 2839 (1971).

30. A. E. Douglas and W. J. Jones, Can. J. Phys. 43, 74 (1965).

31. R. N. Dixon, Phil. Trans. Roy. Soc. 252A, 165 (1960). 